\title{
Prognostic analysis of radical resection for intrahepatic cholangiocarcinoma: a retrospective cohort study
}

\author{
Qingqiang Ni,2, Weifeng Shen², Minfeng Zhang², Cheng Yang², Wenchang Cai², \\ Mengchao $\mathbf{W u}^{2}$ and Jiamei Yang ${ }^{2}$ \\ ${ }^{1}$ Medical College of Soochow University, Suzhou, Jiangsu, China \\ ${ }^{2}$ Department of Special Treatment and Liver Transplantation, Eastern Hepatobiliary Surgery Hospital, Second Military Medical \\ University, Shanghai, China \\ Correspondence to: Weifeng Shen, email: docshenweifeng@163.com \\ Jiamei Yang, email: JMYANG@smmu.edu.cn \\ Keywords: intrahepatic cholangiocarcinoma, radical resection, disease-free survival, overall survival, retrospective cohort study \\ Received: June 21, $2016 \quad$ Accepted: March 09, $2017 \quad$ Published: June 13, 2017
}

Copyright: Ni et al. This is an open-access article distributed under the terms of the Creative Commons Attribution License 3.0 (CC BY 3.0), which permits unrestricted use, distribution, and reproduction in any medium, provided the original author and source are credited.

\section{ABSTRACT}

The aim of this study was to investigate the relationship between the clinicopathological characteristics of intrahepatic cholangiocarcinoma (ICC) and both disease-free survival (DFS) and overall survival (OS) in intrahepatic cholangiocarcinoma (ICC) patients who underwent radical resection (RO). We retrospectively analyzed the clinicopathological characteristics of 319 patients who underwent radical resection of ICC between October 1999 and December 2003. The independent adverse prognostic factors that affected DFS after radical resection of ICC were as follows: maximum tumor diameter $(H R=1.330, P=0.014)$, complicated bile duct stone $(H R=1.923, P=0.013)$, macroscopic tumor thrombus $(H R=1.826$, $P=0.009)$, and lymph node metastasis (Pathology N1) $(H R=2.330, P=0.005)$ were independent adverse prognostic factors that affected the DFS after radical resection of ICC. The postoperative median DFS was 6 months. The independent adverse prognostic factors that affected OS after radical resection of ICC were as follows: maximum tumor diameter $(H R=1.326, P=0.014)$, complicated bile duct stone (HR = 2.349, $P=0.001)$, and lymph node metastasis (Pathology N1) (HR = 2.420, $P=0.003$ ). The postoperative median survival time was 22 months, the 3 -year survival rate was $33.9 \%$, and the 5 -year survival rate was $23.2 \%$. Macroscopic tumor thrombus ( $O R=2.991, P=0.004)$ was an independent risk factor for death within 1 year after radical resection.

\section{INTRODUCTION}

Globally, primary liver carcinoma (PLC) is the fifth most common malignant tumor, resulting in 600,000 deaths each year [1]. Intrahepatic cholangiocarcinoma (ICC) is a carcinoma arising from the epithelial cells of the intrahepatic bile duct branches of the second-order or beyond. Although ICC is rare in clinical practice, its incidence and mortality rates have increased gradually in the past 30 years [2-7]. The incidence of ICC accounts for $10 \%$ of all cholangiocarcinomas and $3 \%$ of gastrointestinal carcinomas $[8,9]$.
ICC is caused by a variety of etiologies, such as hepatitis B, hepatitis C, AIDS, intrahepatic bile duct stones, liver cirrhosis, primary sclerosing cholangitis, parasitic infections, chemical carcinogens, obesity, type II diabetes, and nonalcoholic fatty liver disease [10]. With the continuous improvement of the quality of life in China, the incidences of excessive alcohol consumption, obesity, type II diabetes, and nonalcoholic fatty liver disease have increased. As early as 2006, the number of patients with chronic hepatitis B in China had reached 20 million [11]. The abovementioned factors may be responsible for the world's highest incidence of ICC being reported in Asia, 
including in China [12]. The median survival time in ICC patients is as low as 2-6 months if only palliative treatment is involved. Moreover, the survival time is over 5 years in less than $10 \%$ of patients, regardless of the treatment regimen $[3,13,14]$. However, the 5-year survival rate after surgical resection ranges from $30-35 \%$ [15]. Although hepatectomy is the main treatment method for early ICC, only approximately one-third of ICC patients have an opportunity to undergo resection. The median survival time is only approximately 3 years, and the recurrence rate is as high as 50-60\%; at the time of ICC diagnosis, the majority of patients are in the late stage and missed their opportunity to receive radical resection [16]. A high grade of malignancy, lower radical resection rate, and poor prognosis have resulted in a tremendous effect on ICC patients; therefore, this topic is worth further study.

With an increase in its aging population, China now has a high incidence of ICC, which deserves our full attention. Therefore, this retrospective cohort study was designed to determine the prognostic factors that can affect postoperative disease-free survival (DFS) and overall survival (OS) according to survival data and clinicopathological characteristics in the follow-up of 319 patients who underwent radical resection of ICC. This study aims to provide references for the clinical diagnosis and treatment of ICC.

\section{MATERIALS AND METHODS}

\section{General data}

This single-center, retrospective cohort study was approved by the ethical committee of the Shanghai Eastern Hepatobiliary Surgery Hospital. The study protocol conformed to the principles of the Declaration of Helsinki. The study subjects were selected from 319 patients who underwent radical resection (R0) of ICC from October 1999 to December 2003 in our hospital. Of these patients, 225 were male and 94 were female. Their average age was $52.70 \pm 10.50$ years (range: $22-81$ years). A total of 109 patients ( 76 males and 33 females with a mean age of $53.44 \pm 11.24$ years) were included in the group with $\mathrm{OS} \leq 1$ year. The remaining 210 patients (149 males and 61 females with a mean age of $52.31 \pm 10.10$ years) were included in the group with OS $>1$ year. The balance test showed that there were no significant differences $(\mathrm{P}>$ 0.05 ) in sex or age between the two groups. Thus, the data were compatible. All of the patients were diagnosed with ICC, and their diagnosis was confirmed by pathological examination. Patients with mixed HCC were excluded.

\section{Study methods}

\section{Clinical data collection}

The clinical data of the patients were collected, including gender, age, tumor number, number of liver segments with tumors, perioperative blood transfusion, postoperative pathological type, the presence of liver cirrhosis, maximum tumor diameter, complicated bile duct stone, serum CA199 levels, macroscopic tumor thrombus, and tumor staging. According to their morphology, ICC can be graded as well, moderately and poorly differentiated adenocarcinoma. Well-differentiated ICCs include tubular adenocarcinoma with/without micropapillary structures; moderately differentiated ICCs include moderately distorted tubular glands with cribriform formations and/or a cord-like pattern; and poorly differentiated ICCs include distorted tubular or cord-like structures with marked cellular pleomorphism (WHO classification of tumors of the digestive system) Complicated bile duct stones were defined as the presence of calculi within the intrahepatic biliary tree proximal to the confluence of the left and right hepatic ducts. Tumor staging in all of the patients was determined according to the UICC TNM classification of malignant tumors, 7 th edition.

\section{Definition of radical resection}

Radical hepatectomy primarily includes regular hepatectomy and hemihepatectomy, irregular hepatectomy, extended hepatectomy, and concomitant removal of bile duct stone or regional lymphadenectomy. The following criteria should be met: (1) all tumors are removed without residual tumor; (2) negative margins are confirmed by histological examination; (3) there is no major vascular invasion; and (4) preoperative elevated CA199 levels return to normal within 2 months after surgery. The preoperative assessment of liver function was based on the Child-Pugh scoring system. The surgical method and resection region were determined according to the tumor size and distribution and the hepatic functional reserve. Whether extended hepatectomy was performed was based on an evaluation of the volume of the remaining liver based on the results of a preoperative computed tomography (CT) scan or magnetic resonance imaging (MRI). The clamp crushing and finger fracture methods were used for anatomical liver resection at room temperature, with intermittent use of Pringle's maneuver. The distance between the tumor and cut edge was 1-1.5 $\mathrm{cm}$. The detection of regional lymph node metastases was based on CT/MRI/PET-CT. If regional lymph node metastases are suspected, regional lymphadenectomy should be considered, including complete excision of soft tissue and lymph nodes at the hepatic hilum, common hepatic artery, hepatoduodenal ligament, portal vein and posterior to the pancreatic head stations. A definitive diagnosis was made by pathologic evidence. 


\section{Follow-up}

Follow-up was performed in all of the patients by telephone communication or outpatient visits. The observed outcomes included DFS and OS. The initial event to assess DFS was radical hepatectomy, and the endpoint was the time of tumor recurrence or the occurrence of a censored event. The initial event to assess OS was radical hepatectomy, and the endpoint event was the death of the patient or the occurrence of a censored event. Censored events included the following: (1) loss of patients during follow-up; and (2) no evidence of tumor recurrence or the patient was still alive at the end of the follow-up. Recurrence was diagnosed based on imaging of B-mode ultrasound, CT scan, and MRI, or on confirmation of ICC recurrence by biopsy and pathological examinations. The starting time of follow-up was the time point when radical hepatectomy was performed, and the endpoint was May 15, 2016. Loss of follow-up was defined as the occurrence of the following events: the patient had missed their outpatient visits for 6 months; the patient could not be reached by phone; or the patient was not hospitalized for 6 months. In this study, 118 cases were lost during the follow-up, and 201 cases completed the follow-up. The follow-up rate was $63.01 \%$ (201/319). The follow-up duration ranged between 1 and 177 months, with a median follow-up duration of 13 months.

\section{Statistical analysis}

The statistical analysis was performed with SPSS 17.0 software. The chi-square test was used for the count data, and the rank sum test was used for the ranked data. A multivariate analysis for shorter OS was performed using a binary logistic regression. The survival rate was calculated using the Kaplan-Meier method. The survival rates were compared using the log-rank method. A Cox regression model was used to analyze the prognostic factors. A $\mathrm{P}$ value of less than 0.05 was considered statistically significant.

\section{RESULTS}

\section{Clinicopathological characteristics of the 319} patients who underwent radical resection of ICC

Of the 319 ICC patients, 225 patients were male, and 94 patients were female, with an average age of 52.70 \pm 10.50 years (range: $22-81$ years). A statistical description of the clinicopathological characteristics for these patients is shown in Table 1.

\section{Analysis of the impact factor for DFS after radical resection}

The univariate Cox analysis showed that the number of tumors, tumor distribution, perioperative blood transfusion, maximum tumor diameter, complicated bile duct stone, serum CA199 levels (U/ml), macroscopic tumor thrombus, Pathology T, Pathology N, and pathology stage were the prognostic factors that affected the DFS in patients who underwent radical hepatectomy for ICC ( $P$ $<0.05)$. The significant prognostic factors determined by the univariate analysis were included in a multivariate Cox analysis, which showed that maximum tumor diameter $(\mathrm{HR}=1.330, P=0.014)$, complicated bile duct stone $(\mathrm{HR}=1.923, P=0.013)$, macroscopic tumor thrombus $(\mathrm{HR}=1.826, P=0.009)$, and Pathology N (HR $=2.330$, $P=0.005)$ were independent adverse prognostic factors that affected the DFS of patients who underwent radical hepatectomy for ICC (Table 2). The median DFS was 6 months; the DFS curves are shown in Figure 1A.
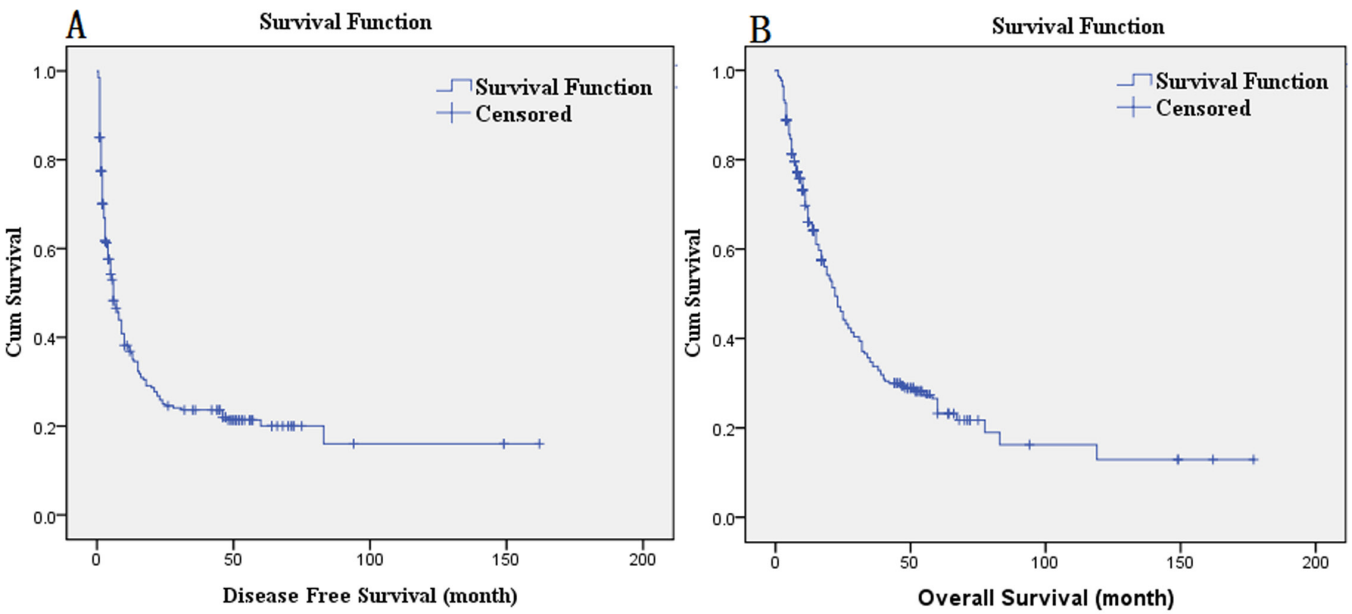

Figure 1: A. DFS curves; B. OS curves. 
Table 1: Clinicopathological characteristics of 319 patients who underwent radical resection of ICC

\begin{tabular}{|c|c|c|c|}
\hline & Number of cases & Total number of cases & Percentage (\%) \\
\hline Gender & & 319 & \\
\hline male & 225 & & 70.5 \\
\hline female & 94 & & 29.5 \\
\hline Age & & 319 & \\
\hline$>55$ years & 126 & & 39.5 \\
\hline$\leq 55$ years & 193 & & 60.5 \\
\hline Single tumor & & 319 & \\
\hline yes & 229 & & 71.8 \\
\hline no & 90 & & 28.2 \\
\hline Tumor distribution & & 319 & \\
\hline$>2$ hepatic segments & 116 & & 36.4 \\
\hline$\leq 2$ hepatic segments & 203 & & 63.6 \\
\hline $\begin{array}{l}\text { Perioperative blood } \\
\text { transfusion }\end{array}$ & & 319 & \\
\hline yes & 137 & & 42.9 \\
\hline no & 182 & & 57.1 \\
\hline Pathological type & & 291 & \\
\hline Well differentiated & 4 & & 1.4 \\
\hline $\begin{array}{l}\text { Moderately } \\
\text { differentiated }\end{array}$ & 113 & & 38.8 \\
\hline Poorly differentiated & 174 & & 59.8 \\
\hline $\begin{array}{l}\begin{array}{l}\text { Evidence of liver } \\
\text { cirrhosis }\end{array} \\
\end{array}$ & & 319 & \\
\hline yes & 123 & & 61.4 \\
\hline no & 196 & & 38.6 \\
\hline \begin{tabular}{ll|}
$\begin{array}{l}\text { Maximum } \\
\text { diameter }(\mathrm{cm})\end{array}$ & tumor \\
\end{tabular} & & 319 & \\
\hline$\leq 5$ & 127 & & 39.8 \\
\hline$>5, \leq 10$ & 139 & & 43.6 \\
\hline$>10$ & 53 & & 16.6 \\
\hline $\begin{array}{l}\text { Complicated bile duct } \\
\text { stone }\end{array}$ & & 318 & \\
\hline yes & 30 & & 9.4 \\
\hline no & 288 & & 90.6 \\
\hline Serum CA199 (U/ml) & & 313 & \\
\hline$>37$ & 174 & & 55.6 \\
\hline$\leq 37$ & 139 & & 44.4 \\
\hline \begin{tabular}{ll|} 
Macroscopic & tumor \\
thrombus & \\
\end{tabular} & & 319 & \\
\hline yes & 39 & & 12.2 \\
\hline no & 280 & & 87.8 \\
\hline Pathology T & & 319 & \\
\hline $\mathrm{T} 1$ & 10 & & 3.1 \\
\hline $\mathrm{T} 2$ & 130 & & 40.8 \\
\hline $\mathrm{T} 3$ & 86 & & 27 \\
\hline $\mathrm{T} 4$ & 93 & & 29.2 \\
\hline Pathology N & & 319 & \\
\hline $\mathrm{N} 0$ & 293 & & 91.8 \\
\hline $\mathrm{N} 1$ & 26 & & 8.2 \\
\hline Pathology M & & 319 & \\
\hline M0 & 316 & & 99.1 \\
\hline M1 & 3 & & 0.9 \\
\hline Pathology stage & & 319 & \\
\hline $\mathrm{I}$ & 10 & & 3.1 \\
\hline II & 119 & & 37.3 \\
\hline III & 97 & & 30.4 \\
\hline IV & 93 & & 29.2 \\
\hline
\end{tabular}


Table 2: Analysis of impact on DFS after radical resection

\begin{tabular}{|c|c|c|c|c|c|c|c|}
\hline \multirow{2}{*}{$\begin{array}{l}\text { Clinicopathological } \\
\text { characteristics }\end{array}$} & \multirow{2}{*}{ Number of cases } & \multicolumn{3}{|c|}{ Univariate analysis } & \multicolumn{3}{|c|}{ Multivariate analysis } \\
\hline & & HR & $P$ & $95 \% \mathrm{CI}$ & HR & $P$ & $95 \%$ CI \\
\hline \multicolumn{2}{|l|}{ Gender } & \multirow{3}{*}{0.917} & \multirow{3}{*}{0.576} & \multirow{3}{*}{$(0.676,1.244)$} & & & \\
\hline male & 225 & & & & & & \\
\hline female & 94 & & & & & & \\
\hline \multicolumn{2}{|l|}{ Age } & \multirow{3}{*}{1.264} & \multirow{3}{*}{0.106} & \multirow{3}{*}{$(0.951,1.680)$} & & & \\
\hline$>55$ years & 126 & & & & & & \\
\hline$\leq 55$ years & 193 & & & & & & \\
\hline \multicolumn{2}{|l|}{ Single tumor } & \multirow{3}{*}{1.621} & \multirow{3}{*}{0.002} & \multirow{3}{*}{$(1.193,2.202)$} & \multirow{3}{*}{1.022} & \multirow{3}{*}{0.948} & \multirow{3}{*}{$(0.538,1.940)$} \\
\hline yes & 229 & & & & & & \\
\hline no & 90 & & & & & & \\
\hline Tumor distribution & & & & & & & \\
\hline$>2$ hepatic segments & 116 & 1.222 & 0.002 & $(1.079,1.348)$ & 1.511 & 0.226 & $(0.775,2.945)$ \\
\hline$\leq 2$ hepatic segments & 203 & & & & & & \\
\hline Perioperative blood transfi & & & & & & & \\
\hline yes & 137 & 1.410 & 0.019 & $(1.059,1.877)$ & 0.998 & 0.993 & $(0.698,1.427)$ \\
\hline no & 182 & & & & & & \\
\hline Pathological type & & & & & & & \\
\hline Well differentiated & 4 & & & & & & \\
\hline Moderately differentiated & 113 & 1.082 & 0.593 & $(0.810,1.445)$ & & & \\
\hline Poorly differentiated & 174 & & & & & & \\
\hline Evidence of liver cirrhosis & & & & & & & \\
\hline yes & 123 & 0.913 & 0.535 & $(0.683,1.219)$ & & & \\
\hline no & 196 & & & & & & \\
\hline Maximum tumor diameter & & & & & & & \\
\hline$\leq 5$ & 127 & 1447 & 0000 & $\left(\begin{array}{llll}1 & 180 & 1 & 761\end{array}\right.$ & 1330 & 0014 & $(10601660)$ \\
\hline$>5, \leq 10$ & 139 & $1.44 /$ & 0.000 & $(1.189,1.101)$ & 1.500 & 0.014 & $(1.000,1.009)$ \\
\hline$>10$ & 53 & & & & & & \\
\hline Complicated bile duct ston & & & & & & & \\
\hline yes & 30 & 1.718 & 0.020 & $(1.089,2.708)$ & 1.923 & 0.013 & $(1.145,3.227)$ \\
\hline no & 288 & & & & & & \\
\hline Serum CA199 (U/ml) & & & & & & & \\
\hline$>37$ & 174 & 1.456 & 0.011 & $(1.089,1.946)$ & 1.266 & 0.486 & $(0.652,2.457)$ \\
\hline$\leq 37$ & 139 & & & & & & \\
\hline Macroscopic tumor throml & & & & & & & \\
\hline yes & 39 & 1.739 & 0.009 & $(1.146,2.637)$ & 1.826 & 0.009 & $(1.160,2.873)$ \\
\hline no & 280 & & & & & & \\
\hline Pathology T & & & & & & & \\
\hline $\mathrm{T} 1$ & 10 & & & & & & \\
\hline $\mathrm{T} 2$ & 130 & 1.363 & 0.000 & $(1.158,1.604)$ & 1.727 & 0.091 & $(0.917,3.251)$ \\
\hline T3 & 86 & & & & & & \\
\hline $\mathrm{T} 4$ & 93 & & & & & & \\
\hline Pathology N & & & & & & & \\
\hline N0 & 293 & 2.156 & 0.005 & $(1.261,3.686)$ & 2.330 & 0.005 & $(1.297,4.186)$ \\
\hline N1 & 26 & & & & & & \\
\hline Pathology M & & & & & & & \\
\hline M0 & 316 & 1.245 & 0.759 & $(0.308,5.028)$ & & & \\
\hline M1 & 3 & & & & & & \\
\hline Pathology stage & & & & & & & \\
\hline $\mathrm{I}$ & 10 & & & & & & \\
\hline II & 119 & 1.383 & 0.000 & $(1.172,1.630)$ & 0.986 & 0.972 & $(0.434,2.239)$ \\
\hline III & 97 & & & & & & \\
\hline IV & 93 & & & & & & \\
\hline
\end{tabular}


Table 3: Analysis of impact on OS after radical resection

\begin{tabular}{|c|c|c|c|c|c|c|c|}
\hline \multirow{2}{*}{$\begin{array}{l}\text { Clinicopathological } \\
\text { characteristics }\end{array}$} & \multirow{2}{*}{$\begin{array}{l}\text { Number of } \\
\text { cases }\end{array}$} & \multicolumn{3}{|c|}{ Univariate analysis } & \multicolumn{3}{|c|}{ Multivariate analysis } \\
\hline & & HR & $P$ & $95 \% \mathrm{CI}$ & HR & $P$ & $95 \% \mathrm{CI}$ \\
\hline Gender & & \multirow{3}{*}{0.915} & \multirow{3}{*}{0.566} & \multirow{3}{*}{$(0.675,1.240)$} & & & \\
\hline male & 225 & & & & & & \\
\hline female & 94 & & & & & & \\
\hline Age & & \multirow{3}{*}{1.193} & \multirow{3}{*}{0.229} & \multirow{3}{*}{$(0.895,1.589)$} & & & \\
\hline$>55$ years & 126 & & & & & & \\
\hline$\leq 55$ years & 193 & & & & & & \\
\hline \multicolumn{2}{|l|}{ Single tumor } & \multirow{3}{*}{1.267} & \multirow{3}{*}{0.132} & \multirow{3}{*}{$(0.932,1.723)$} & & & \\
\hline yes & 229 & & & & & & \\
\hline no & 90 & & & & & & \\
\hline \multicolumn{2}{|l|}{ Tumor distribution } & \multirow{3}{*}{1.710} & \multirow{3}{*}{0.000} & \multirow{3}{*}{$(1.272,2.299)$} & \multirow{3}{*}{1.209} & & \\
\hline$>2$ hepatic segments & 116 & & & & & 0.331 & $(0.825$ \\
\hline$\leq 2$ hepatic segments & 203 & & & & & & \\
\hline Perioperative blood transf & & & & & & & \\
\hline yes & 137 & 1274 & 0.097 & $(0.957 .1697)$ & & & \\
\hline no & 182 & & & & & & \\
\hline Pathological type & & & & & & & \\
\hline Well differentiated & 4 & & & & & & \\
\hline Moderately differentiated & 113 & 1.173 & 0.272 & $(0.882,1.560)$ & & & \\
\hline Poorly differentiated & 174 & & & & & & \\
\hline Evidence of liver cirrhosis & & & & & & & \\
\hline yes & 123 & 0.888 & 0.424 & $(0.662,1.189)$ & & & \\
\hline no & 196 & & & & & & \\
\hline Maximum tumor diameter & & & & & & & \\
\hline$\leq 5$ & 127 & & & & & & \\
\hline$>5, \leq 10$ & 139 & 1.451 & 0.000 & $(1.190,1.770)$ & 1.326 & 0.014 & $\begin{array}{c}(1.059, \\
1.660)\end{array}$ \\
\hline$>10$ & 53 & & & & & & \\
\hline Complicated bile duct stor & & & & & & & \\
\hline yes & 30 & 1874 & 0.007 & $(1.187 .2960)$ & 2349 & 0.001 & $(1.397$, \\
\hline no & 288 & & & & & & $3.950)$ \\
\hline Serum CA199 (U/ml) & & & & & & & \\
\hline$>37$ & 174 & 1494 & 0007 & $(1.115,2001)$ & 1.178 & 0309 & $(0.859$ \\
\hline$\leq 37$ & 139 & & & $(1.11 J, 2.001)$ & & & 1.617) \\
\hline Macroscopic tumor throm & & & & & & & \\
\hline yes & 39 & 1.422 & 0.109 & $(0.925,2.187)$ & & & \\
\hline no & 280 & & & & & & \\
\hline Pathology T & & & & & & & \\
\hline $\mathrm{T} 1$ & 10 & & & & & & \\
\hline $\mathrm{T} 2$ & 130 & 1272 & 0004 & (1080 1498) & 0636 & 1248 & $(0.499$ \\
\hline $\mathrm{T} 3$ & 86 & & & & & & $3.125)$ \\
\hline $\mathrm{T} 4$ & 93 & & & & & & \\
\hline Pathology N & & & & & & & \\
\hline N1 & 26 & 2578 & 0.001 & $(1.491 .4458)$ & 2420 & 0.003 & $(1.340$ \\
\hline N0 & 293 & & & & & & 4.372) \\
\hline Pathology M & & & & & & & \\
\hline M0 & 316 & 1588 & 0516 & $(0393 \quad 6413)$ & & & \\
\hline M1 & 3 & & & & & & \\
\hline Pathology stage & & & & & & & \\
\hline $\mathrm{I}$ & 10 & & & & & & \\
\hline II & 119 & 1299 & 0002 & (1.101, & 0912 & 0.949 & $(0.375$, \\
\hline III & 97 & & & $1.532)$ & & & 2.398) \\
\hline IV & 93 & & & & & & \\
\hline
\end{tabular}




\section{Impact factor analysis of OS after radical resection}

The univariate Cox analysis showed that the tumor distribution, maximum tumor diameter, complicated bile duct stone, serum CA199 level (U/ml), Pathology T, Pathology N, and pathology stage were the prognostic factors for $\mathrm{OS}$ in patients who underwent radical hepatectomy for $\operatorname{ICC}(P<0.05)$. The significant prognostic factors determined by the univariate analysis were included in the multivariate Cox analysis, which showed that the maximum tumor diameter $(\mathrm{HR}=1.326$, $P=0.014)$, complicated bile duct stone (HR $=2.349$, $P=0.001)$, and Pathology N $(\mathrm{HR}=2.420, P=0.003)$ were independent adverse prognostic factors that affected OS of patients who underwent radical hepatectomy for ICC (Table 3). The postoperative median survival time was 22 months; the 3-year survival rate was 33.9\%; and the 5-year survival rate was $23.2 \%$. The OS curves are shown in Figure 1B. The survival comparison by logrank method of independent prognostic factors, such as maximum tumor diameter, complicated bile duct stone, and Pathology N, are shown in Figure 2.

\section{Risk factor analysis for shorter $\mathrm{OS}(\mathrm{OS} \leq 1$ year) after radical resection}

We defined shorter OS as OS $\leq 1$ year. A total of 109 patients (76 males and 33 females with a mean age of $53.44 \pm 11.24$ years) were included in the group with OS $\leq 1$ year. The remaining 210 patients (149 males and 61 females with a mean age of $52.31 \pm 10.10$ years) were included in the group with OS $>1$ year. The balance test showed that there was no significant difference $(\mathrm{P}>0.05)$ in sex or age between the 2 groups. Thus, the data were compatible. The univariate analysis showed that the tumor number, tumor distribution, complicated bile duct stone, macroscopic tumor thrombus, and Pathology $\mathrm{T}$ may be relevant factors that result in a postoperative shorter OS $(P$ $<0.05)$. The binary logistic multivariate analysis showed that macroscopic tumor thrombus may be an independent risk factor for a shorter OS in ICC patients after radical resection $(\mathrm{OR}=2.991, P=0.004)($ Table 4$)$.

\section{DISCUSSION}

The prognostic factors for survival in ICC patients have not been fully elucidated, and many factors and mechanisms remain under investigation. In this study, follow-up (duration: 1-177 months) for survival was performed in 319 patients who underwent radical hepatectomy of ICC between October 1999 and December 2003 in our hospital. The median follow-up duration was 13 months; 118 cases were lost during the follow-up. The possible prognostic factors related to ICC were analyzed, and the high risk factors for poor prognosis in ICC patients were investigated.

In this study, a multivariate Cox analysis showed that the maximum tumor diameter, complicated bile duct stone, and lymph node metastasis were independent adverse prognostic factors that affected the DFS and OS in ICC patients after radical hepatectomy. Macroscopic tumor thrombus is an independent adverse prognostic factor for DFS in ICC patients after radical hepatectomy. Whether the tumor diameter affects survival time after hepatectomy has been controversial. The 6th edition of the AJCC TNM staging system showed that the presence of an isolated lesion or multiple lesions with a diameter $\leq 5 \mathrm{~cm}$ indicate a good prognosis. However, the staging system proposed by Okabayashi and Nathan and the 7th edition of the AJCC TNM staging system suggested that tumor diameter is not an independent prognostic factor for postoperative survival time. More recently, there are data to show that size does play a role up to a maximum size of $7 \mathrm{~cm}$ and then plateaus. Yamasaki suggested that ICC patients with tumors $>2 \mathrm{~cm}$ in diameter had a relatively poor prognosis [17]. This study has demonstrated that maximum tumor diameter was an independent adverse prognostic factor for survival time after radical resection of ICC. When the maximum tumor diameter increased by one level, the risk of recurrence and death increased 1.330
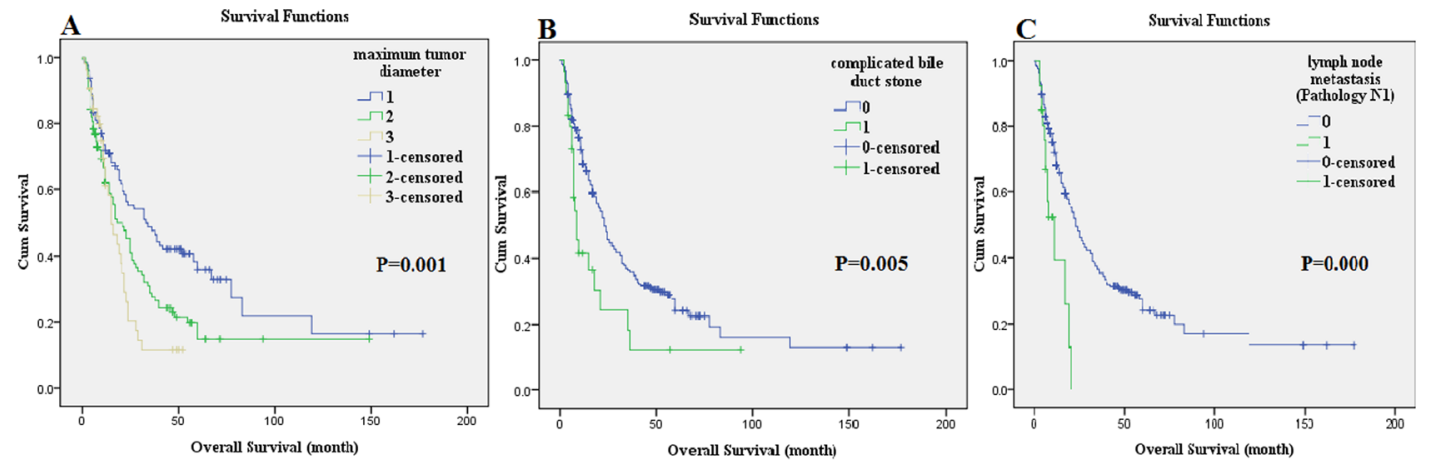

Figure 2: The survival comparison of independent prognostic factors using the log-rank method: maximum tumor diameter. A., complicated bile duct stone B., and lymph node metastasis (Pathology N1) C. 
Table 4: Analysis of risk on shorter $\mathrm{OS}(\mathrm{OS} \leq 1$ year) after radical resection

\begin{tabular}{|c|c|c|c|c|c|c|c|}
\hline \multirow[b]{2}{*}{ Clinicopathological characteristics } & \multirow{2}{*}{$\begin{array}{l}\text { Number of } \\
\text { cases }\end{array}$} & \multicolumn{3}{|c|}{ Univariate analysis } & \multicolumn{3}{|c|}{ Multivariate analysis } \\
\hline & & $\begin{array}{l}\text { Group with } \\
\text { OS } \leq 1 \text { year }\end{array}$ & \begin{tabular}{|l|} 
Group with \\
OS $>1$ year
\end{tabular} & $P$ & OR & $P$ & $95 \% \mathrm{CI}$ \\
\hline Gender & & & & \multirow{3}{*}{0.820} & & & \\
\hline male & 225 & 76 & 149 & & & & \\
\hline female & 94 & 33 & 61 & & & & \\
\hline \multicolumn{4}{|l|}{ Age } & \multirow{3}{*}{0.477} & & & \\
\hline$>55$ years & 126 & 46 & 80 & & & & \\
\hline$\leq 55$ years & 193 & 63 & 130 & & & & \\
\hline \multicolumn{4}{|l|}{ Single tumor } & \multirow{3}{*}{0.031} & \multirow{3}{*}{0.748} & \multirow{3}{*}{0.568} & \multirow{3}{*}{$(0.275,2.029)$} \\
\hline yes & 229 & 70 & 159 & & & & \\
\hline no & 90 & 39 & 51 & & & & \\
\hline \multicolumn{4}{|l|}{ Tumor distribution } & \multirow{3}{*}{0.011} & \multirow{3}{*}{1.661} & \multirow{3}{*}{0.57} & \multirow{3}{*}{$(0.986,2.799)$} \\
\hline$>2$ hepatic segments & 116 & 50 & 66 & & & & \\
\hline$\leq 2$ hepatic segments & 203 & 59 & 144 & & & & \\
\hline \multicolumn{4}{|l|}{ Perioperative blood transfusion } & \multirow{3}{*}{0.216} & & & \\
\hline yes & 137 & 52 & 85 & & & & \\
\hline no & 182 & 57 & 125 & & & & \\
\hline Pathological type & & & & & & & \\
\hline Well differentiated & 4 & 1 & 3 & & & & \\
\hline Moderately differentiated & 113 & 32 & 81 & 0.160 & & & \\
\hline Poorly differentiated & 174 & 68 & 106 & & & & \\
\hline Evidence of liver cirrhosis & & & & & & & \\
\hline yes & 123 & 42 & 81 & 0.995 & & & \\
\hline no & 196 & 67 & 129 & & & & \\
\hline Maximum tumor diameter $(\mathrm{cm})$ & & & & & & & \\
\hline$\leq 5$ & 127 & & & & & & \\
\hline$>5, \leq 10$ & 139 & 109 & 210 & 0.071 & & & \\
\hline$>10$ & 53 & 更 & 210 & & & & \\
\hline Complicated bile duct stone & & & & & & & \\
\hline yes & 30 & 16 & 14 & 0021 & 2755 & 0232 & $(1496605)$ \\
\hline no & 288 & 93 & 195 & & & & \\
\hline Serum CA199 (U/ml) & & & & & & & \\
\hline$>37$ & 174 & 68 & 106 & 0.077 & & & \\
\hline$\leq 37$ & 139 & 41 & 98 & 0.011 & & & \\
\hline Macroscopic tumor thrombus & & & & & & & \\
\hline yes & 39 & 23 & 16 & 0.000 & 2.991 & 0.004 & $(1.429,6.260)$ \\
\hline no & 280 & 86 & 194 & & & & \\
\hline Pathology T & & & & & & & \\
\hline T1 & 10 & & & & & & \\
\hline $\mathrm{T} 2$ & 130 & & & 0000 & 1237 & 0324 & $(08101800)$ \\
\hline T3 & 86 & 109 & 210 & 0.000 & 1.231 & 0.324 & $(0.810,1.890)$ \\
\hline $\mathrm{T} 4$ & 93 & & & & & & \\
\hline Pathology N & & & & & & & \\
\hline N0 & 293 & 100 & 210 & 0.362 & & & \\
\hline N1 & 26 & 109 & 210 & & & & \\
\hline Pathology M & & & & & & & \\
\hline M0 & 316 & 100 & | & 0211 & & & \\
\hline M1 & 3 & 109 & 210 & 0.211 & & & \\
\hline Pathology stage & & & & & & & \\
\hline $\mathrm{I}$ & 10 & & & & & & \\
\hline II & 119 & & & 0.057 & & & \\
\hline III & 97 & 109 & 210 & & & & \\
\hline IV & 93 & & & & & & \\
\hline
\end{tabular}


and 1.326 times, respectively. A study by Nordenstedt showed that gallstones can increase the risk of intrahepatic and extrahepatic cholangiocarcinoma [18]. Mechanisms of intrahepatic bile duct stone formation remain unclear and may be associated with malnutrition, low economic status, and biliary tract infections. Intrahepatic bile duct stones are closely correlated with the incidence of ICC and therefore may be related to the recurrence of ICC after radical resection [19-21]. In this study, the multivariate analysis showed that complicated bile duct stones are an independent adverse prognostic factor that can affect DFS in patients after radical resection of ICC. The impact of lymph node metastasis on postoperative survival in ICC patients remains debatable. The effect of intraoperative lymphadenectomy during the resection of ICC on prognosis is not yet supported by sufficient data. A study by Shimada et al. showed that intraoperative lymphadenectomy does not improve the prognosis of ICC patients [22]. Studies by Morine et al. have shown that prophylactic lymphadenectomy is not necessary for ICC patients who do not display lymph node metastasis [23]. The study by Li et al. showed that ICC patients without evidence of lymph node metastasis and ICC patients with multiple tumors and lymph node metastasis did not benefit from lymphadenectomy [24]. A systematic review by Amini et al. showed that the 3- and 5-year survival rates were only 0.2 and $0 \%$, respectively, in ICC patients and lymph node metastasis. Although there are not sufficient data to support intraoperative routine lymphadenectomy, lymphadenectomy is recommended during the resection of ICC because of the higher rate of lymph node metastasis in ICC patients [25]. Some studies have shown that lymph node metastasis is an independent prognostic factor for postoperative survival in ICC patients [26-28]. This study also confirmed that lymph node metastasis was an independent adverse prognostic factor that affects DFS in patients after radical resection of ICC. Terada et al. have reported one case of portal hypertension caused by extensive portal vein tumor thrombi; that patient died of liver function failure 50 days after hospitalization [29]. This study indicated that the macroscopic tumor thrombus was an independent adverse prognostic factor for DFS in patients after undergoing radical resection of ICC. The study by Farges et al. suggested that a satellite lesion was a prognostic factor for OS in patients after undergoing radical resection of ICC; however, these authors were not able to demonstrate that a satellite lesion was an independent prognostic factor for OS in ICC patients [30]. In this group of patients, the postoperative median survival time was 22 months, the 3-year survival rate was $33.9 \%$, and the 5 -year survival rate was $23.2 \%$. The 5 -year survival rate was higher than that reported by Lang et al., Nathan et al., Jan et al., Ohtsuka et al., and Weimann et al. [31-35].

In a comparison between the group with $\mathrm{OS} \leq 1$ year (shorter OS) and the group with OS $>1$ year, the univariate analysis showed that multiple tumors, tumor distribution, complicated bile duct stone, macroscopic tumor thrombus, and lymph node metastasis were possible risk factors for the shorter OS. The studies by Michael et al. revealed that the following factors led to a shorter OS: age, large size of tumor, multiple tumors, lymph node metastasis, vascular invasion, and poorly differentiated tumors [36]. In this study, the univariate analysis indicated that multiple tumors and lymph node metastasis are risk factors for shorter OS. These results are consistent with the findings of Michael et al. In the present study, significant risk factors identified by the univariate analysis were further included in the binary logistic multivariate analysis, which confirmed that macroscopic tumor thrombus may be an independent risk factor for a shorter OS. Studies by Zou et al. have shown that ARID1A, PBRM1, and BAP1, which are tumor suppressor genes, played an important role in suppressing mutations and preventing the occurrence of ICC. The mutation of any single gene in the three wildtype genes can lead to a shorter survival time [37]. This study provided references for further investigating the risk factors of shorter OS after radical resection of ICC.

In summary, radical resection is the only method to potentially cure ICC. However, most patients miss their opportunity for radical surgery because of the late stage of the disease at the time of diagnosis. Even when radical resection is performed, the high recurrence rate and short survival time can negatively affect both the clinicians and ICC patients. In this study, the multiyear follow-up demonstrated that the maximum tumor diameter, complicated bile duct stone, macroscopic tumor thrombus, and lymph node metastasis are independent adverse prognostic factors that can affect survival time in patients who undergo radical resection of ICC. Moreover, risk factors for shorter OS were analyzed. The results showed that macroscopic tumor thrombus is possibly an independent risk factor for mortality within 1 year in patients who undergo radical resection of ICC. The results of this study are intended to guide clinicians in identifying patients with high risks and to give appropriate treatments and interventions as early as possible to improve the life quality and postoperative survival rate of patients.

\section{Author contributions}

Qingqiang Ni analyzed data and wrote the manuscript. Weifeng Shen acquired data. Minfeng Zhang, Cheng Yang and Wenchang Cai assisted in editing the manuscript. Weifeng Shen, Mengchao Wu and Jiamei Yang contributed to the design of the study. Weifeng Shen and Jiamei Yang are the corresponding authors of the paper. All authors read and approved the final manuscript. 


\section{ETHICAL APPROVAL}

This single-center retrospective cohort study was approved by the ethical committee of the Shanghai Eastern Hepatobiliary Surgery Hospital.

\section{CONFLICTS OF INTEREST}

No benefits in any form have been received or will be received from a commercial party related directly or indirectly to the subject of this article.

\section{REFERENCES}

1. Ferlay J, Shin HR, Bray F, Forman D, Mathers C, Parkin DM. Estimates of worldwide burden of cancer in 2008: GLOBOCAN 2008. Int J Cancer. 2010; 127:2893-917.

2. Khan SA, Toledano MB, Taylor-Robinson SD. Epidemiology, risk factors, and pathogenesis of cholangiocarcinoma. HPB (Oxford). 2008; 10:77-82.

3. Shaib Y, El-Serag HB. The epidemiology of cholangiocarcinoma. Semin Liver Dis. 2004; 24:115-25.

4. Patel T. Increasing incidence and mortality of primary intrahepatic cholangiocarcinoma in the United States. Hepatology. 2001; 33:1353-57.

5. Patel T. Worldwide trends in mortality from biliary tract malignancies. BMC Cancer. 2002; 2:10.

6. Poultsides GA, Zhu AX, Choti MA, Pawlik TM. Intrahepatic cholangiocarcinoma. Surg Clin North Am. 2010; 90:817-37.

7. Aljiffry M, Abdulelah A, Walsh M, Peltekian K, Alwayn I, Molinari M. Evidence-based approach to cholangiocarcinoma: a systematic review of the current literature. J Am Coll Surg. 2009; 208:134-47.

8. DeOliveira ML, Cunningham SC, Cameron JL, Kamangar F, Winter JM, Lillemoe KD, Choti MA, Yeo CJ, Schulick RD. Cholangiocarcinoma: thirty-one-year experience with 564 patients at a single institution. Ann Surg. 2007; 245:755-62.

9. Razumilava N, Gores GJ. Cholangiocarcinoma. Lancet. 2014; 383:2168-79.

10. Lafaro KJ, Cosgrove D, Geschwind JF, Kamel I, Herman JM, and Pawlik TM. Multidisciplinary Care of Patients with Intrahepatic Cholangiocarcinoma: Updates in Management. Gastroenterol Res Pract. 2015; 2015: 860861.

11. Lu FM, Zhuang H. Management of hepatitis B in China. Chin Med J (Engl). 2009; 122:3-4.

12. Lim JH, Park CK. Pathology of cholangiocarcinoma. Abdom Imaging. 2004; 29:540-47.

13. Park J, Kim MH, Kim KP, Park DH, Moon SH, Song TJ, Eum J, Lee SS, Seo DW, Lee SK. Natural History and Prognostic Factors of Advanced Cholangiocarcinoma without Surgery, Chemotherapy, or Radiotherapy: A LargeScale Observational Study. Gut Liver. 2009; 3:298-305.
14. Farley DR, Weaver AL, Nagorney DM. "Natural history" of unresected cholangiocarcinoma: patient outcome after noncurative intervention. Mayo Clin Proc. 1995; 70:42529.

15. de Jong MC, Nathan H, Sotiropoulos GC, Paul A, Alexandrescu S, Marques H, Pulitano C, Barroso E, Clary BM, Aldrighetti L, Ferrone CR, Zhu AX, Bauer TW, et al. Intrahepatic cholangiocarcinoma: an international multiinstitutional analysis of prognostic factors and lymph node assessment. J Clin Oncol. 2011; 29:3140-45.

16. Moeini A, Sia D, Bardeesy N, Mazzaferro V, Llovet JM. Molecular Pathogenesis and Targeted Therapies for Intrahepatic Cholangiocarcinoma. Clin Cancer Res. 2016; 22:291-300.

17. Yamasaki S. Intrahepatic cholangiocarcinoma: macroscopic type and stage classification. J Hepatobiliary Pancreat Surg. 2003; 10:288-91.

18. Nordenstedt H, Mattsson F, El-Serag H, Lagergren J. Gallstones and cholecystectomy in relation to risk of intraand extrahepatic cholangiocarcinoma. Br J Cancer. 2012; 106:1011-15.

19. Wen CC, Lee HC. Intrahepatic stones: a clinical study. Ann Surg. 1972; 175:166-77.

20. Kubo S, Kinoshita H, Hirohashi K, Hamba H. Hepatolithiasis associated with cholangiocarcinoma. World J Surg. 1995; 19:637-41.

21. Donato F, Gelatti U, Tagger A, Favret M, Ribero ML, Callea F, Martelli C, Savio A, Trevisi P, Nardi G. Intrahepatic cholangiocarcinoma and hepatitis $\mathrm{C}$ and B virus infection, alcohol intake, and hepatolithiasis: a case-control study in Italy. Cancer Causes Control. 2001; 12:959-64.

22. Shimada M, Yamashita Y, Aishima S, Shirabe K, Takenaka $\mathrm{K}$, Sugimachi K. Value of lymph node dissection during resection of intrahepatic cholangiocarcinoma. Br J Surg. 2001; 88:1463-66.

23. Morine Y, Shimada M. The value of systematic lymph node dissection for intrahepatic cholangiocarcinoma from the viewpoint of liver lymphatics. J Gastroenterol. 2015; 50:913-27.

24. Li DY, Zhang HB, Yang N, Quan Y, Yang GS. Routine lymph node dissection may be not suitable for all intrahepatic cholangiocarcinoma patients: results of a monocentric series. World J Gastroenterol. 2013; 19:908491.

25. Amini N, Ejaz A, Spolverato G, Maithel SK, Kim Y, Pawlik TM. Management of lymph nodes during resection of hepatocellular carcinoma and intrahepatic cholangiocarcinoma: a systematic review. J Gastrointest Surg. 2014; 18:2136-48.

26. Wang Y, Li J, Xia Y, Gong R, Wang K, Yan Z, Wan X, Liu G, Wu D, Shi L, Lau W, Wu M, Shen F. Prognostic nomogram for intrahepatic cholangiocarcinoma after partial hepatectomy. J Clin Oncol. 2013; 31:1188-95. 
27. Ribero D, Pinna AD, Guglielmi A, Ponti A, Nuzzo G, Giulini SM, Aldrighetti L, Calise F, Gerunda GE, Tomatis M, Amisano M, Berloco P, Torzilli G, Capussotti L, and Italian Intrahepatic Cholangiocarcinoma Study Group. Surgical Approach for Long-term Survival of Patients With Intrahepatic Cholangiocarcinoma: A Multi-institutional Analysis of 434 Patients. Arch Surg. 2012; 147:1107-13.

28. Jiang W, Zeng ZC, Tang ZY, Fan J, Sun HC, Zhou J, Zeng MS, Zhang BH, Ji Y, Chen YX. A prognostic scoring system based on clinical features of intrahepatic cholangiocarcinoma: the Fudan score. Ann Oncol. 2011; 22:1644-52.

29. Terada T, Kida T, Nakanuma Y, Noguchi T. Extensive portal tumor thrombi with portal hypertension in an autopsy case of intrahepatic cholangiocarcinoma. Am J Gastroenterol. 1992; 87:1513-18.

30. Farges O, Fuks D, Boleslawski E, Le Treut YP, Castaing D, Laurent A, Ducerf C, Rivoire M, Bachellier P, Chiche L, Nuzzo G, Regimbeau JM. Influence of surgical margins on outcome in patients with intrahepatic cholangiocarcinoma: a multicenter study by the AFC-IHCC-2009 study group. Ann Surg. 2011; 254:824-29.

31. Lang H, Sotiropoulos GC, Sgourakis G, Schmitz KJ, Paul A, Hilgard P, Zöpf T, Trarbach T, Malagó M, Baba HA, Broelsch CE. Operations for intrahepatic cholangiocarcinoma: single-institution experience of 158 patients. J Am Coll Surg. 2009; 208:218-28.
32. Nathan H, Aloia TA, Vauthey JN, Abdalla EK, Zhu AX, Schulick RD, Choti MA, Pawlik TM. A proposed staging system for intrahepatic cholangiocarcinoma. Ann Surg Oncol. 2009; 16:14-22.

33. Jan YY, Yeh CN, Yeh TS, Hwang TL, Chen MF. Clinicopathological factors predicting long-term overall survival after hepatectomy for peripheral cholangiocarcinoma. World J Surg. 2005; 29:894-98.

34. Ohtsuka M, Ito H, Kimura F, Shimizu H, Togawa A, Yoshidome H, Miyazaki M. Results of surgical treatment for intrahepatic cholangiocarcinoma and clinicopathological factors influencing survival. Br J Surg. 2002; 89:1525-31.

35. Weimann A, Varnholt H, Schlitt HJ, Lang H, Flemming P, Hustedt C, Tusch G, Raab R. Retrospective analysis of prognostic factors after liver resection and transplantation for cholangiocellular carcinoma. Br J Surg. 2000; 87:118287.

36. Mavros MN, Economopoulos KP, Alexiou VG, Pawlik TM. Treatment and Prognosis for Patients With Intrahepatic Cholangiocarcinoma: Systematic Review and Metaanalysis. JAMA Surg. 2014; 149:565-74.

37. Zou S, Li J, Zhou H, Frech C, Jiang X, Chu JS, Zhao X, Li Y, Li Q, Wang H, Hu J, Kong G, Wu M, et al. Mutational landscape of intrahepatic cholangiocarcinoma. Nat Commun. 2014; 5:5696. 